\title{
CHARACTERIZATION OF LASER DOPPLER VIBROMETERS USING ACOUSTO-OPTIC MODULATORS
}

\author{
Michael Gaitan ${ }^{1}$, Jon Geist ${ }^{2}$, Benjamin J. Reschovsky ${ }^{3}$, Ako Chijioke ${ }^{4}$ \\ ${ }^{1}$ NIST, Gaithersburg MD, USA, michael.gaitan@nist.gov \\ ${ }^{2}$ NIST, Gaithersburg MD, USA, jon.geist@nist.gov \\ ${ }^{3}$ NIST, Gaithersburg MD, USA, benjamin.reschovsky@ nist.gov \\ ${ }^{4}$ NIST, Gaithersburg MD, USA, akobuije.chijioke@nist.gov
}

\begin{abstract}
:
We report on a new approach to characterize the performance of a laser Doppler vibrometer (LDV). The method uses two acousto-optic modulators (AOMs) to frequency shift the light from an LDV by a known quantity to create a synthetic velocity shift that is traceable to a frequency reference. Results are presented for discrete velocity shifts and for sinusoidal velocity shifts that would be equivalent to what would be observed in an ideal accelerometer vibration calibration. The method also enables the user to sweep the synthetic vibration excitation frequency to characterize the bandwidth of an LDV together with its associated electronics.
\end{abstract}

Keywords: Laser Vibrometer; Vibration Calibration; Acousto-Optic Modulator;

\section{INTRODUCTION}

Following ISO Standard 16063-41 Method for the calibration of vibration and shock transducers Part 41 Calibration of laser vibrometers [1], laser Doppler vibrometers (LDVs) are calibrated by a comparison-type measurement to a laser homodyne interferometer that is defined as the primary standard. Not covered by ISO 16063-41, but for the case where all the components of the LDV system and their associated uncertainties are known, methods can be employed for the direct determination of measurement uncertainty of the LDV [2], [3] or by using a combination of a heterodyne with a homodyne-quadrature configuration [4].

The technology for manufacturing commercial LDV systems has matured as well as their use in commercially available primary vibration calibration systems. These systems require calibration by the manufacturer over a periodic time interval that is typically one year and are traceable to the Système International d'Unités (SI) through the manufacturer. From the end user perspective, the commercially manufactured LDV system is like a "black box", meaning that the design and internal components of the LDV are not known in detail by the user. Therefore, following an uncertainty determination approach described in [2] or [3] is not possible for such commercial black box systems especially if their internal workings are considered proprietary by the manufacturer. The only possibility provided by the standard for calibrating a such commercial LDV systems is therefore to follow ISO 16063-41 and compare it to a primary heterodyne system, resulting in the LDV system being considered a secondary system.

A challenge therefore remains in the adoption of cost-effective commercial LDV systems by National Measurement Institutes (NMIs) who are responsible for direct determination of uncertainty. Towards this end, we have recently reported on the calibration of laser heterodyne velocimeters using shock excitations and total distance travelled [5], [6] One advantage of that method is that it characterizes the entire measurement system under the same conditions that would be used in an accelerometer shock calibration and could as well be included as part of the accelerometer shock calibration. However, a drawback of the method is that it does not characterize the frequency response and bandwidth of the LDV. The bandwidth of the excitation must not exceed the bandwidth of the LDV in order to produce accurate accelerometer shock calibrations. This drawback motivated us to develop a new method to characterize the bandwidth of the LDV system and resulted in the method that we present in this report.

\section{EXPERIMENTAL DESIGN}

Figure 1 shows a diagram and photograph of the acousto-optic modulator (AOM)-based LDV characterization system that we have developed. The laser light is first collimated using $300 \mathrm{~mm}$ and $30 \mathrm{~mm}$ lenses to create a beam diameter that is compatible with the aperture of the AOMs. The 

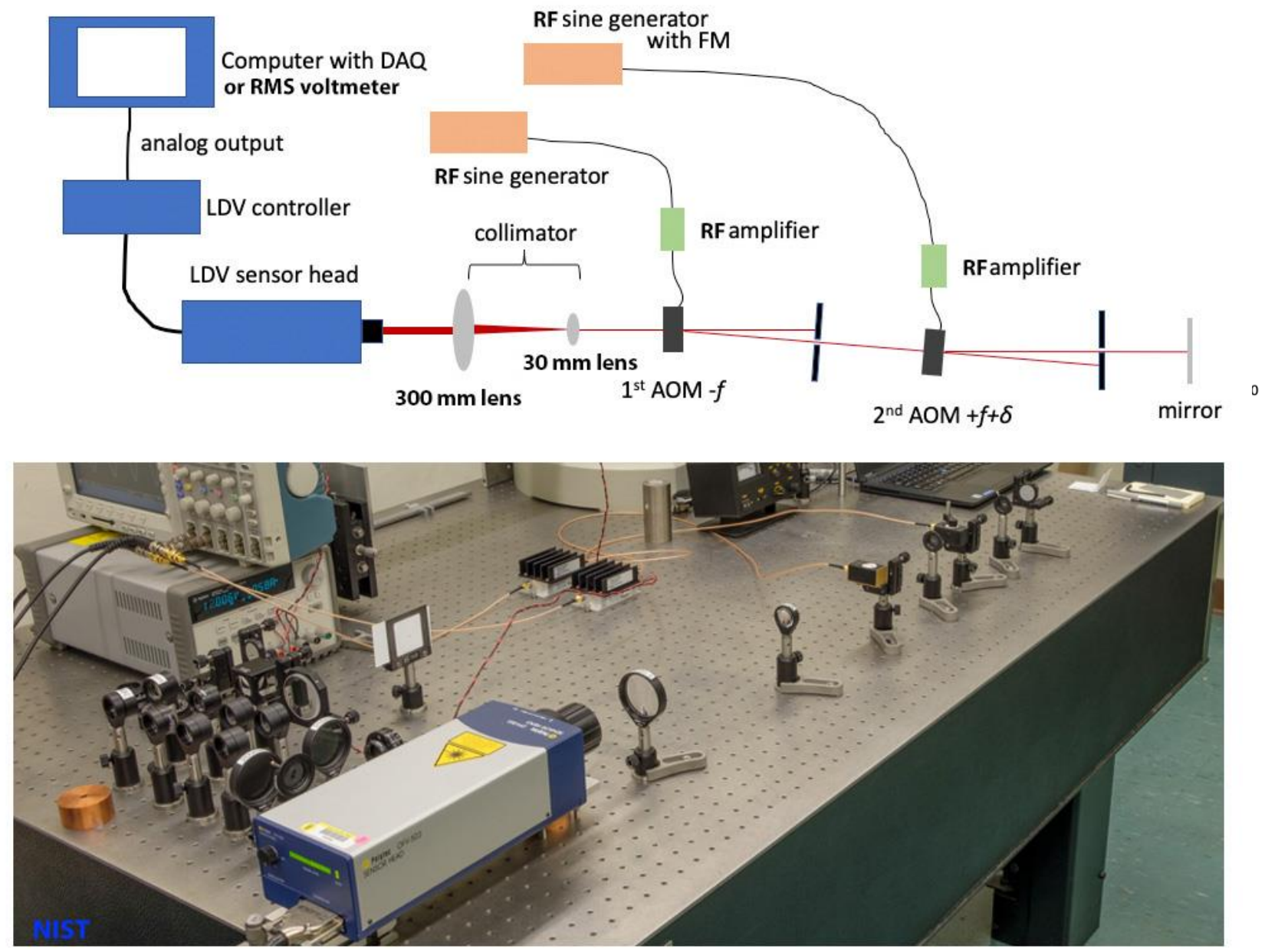

Figure 1: Diagram (upper) and photograph (lower) of the acousto-optic modulator (AOM)-based LDV characterization system.

beam passes through the $1^{\text {st }} \mathrm{AOM}$ where it is downshifted in frequency $f$. Next it passes through the $2^{\text {nd }} A O M$ where it is upshifted by a frequency $f+\delta$. The beam is then reflected back along its path with a mirror, doubling the effect of the AOMs, and delivering light to the LDV that is shifted in frequency by:

$$
2 \delta=2(f+\delta-f)
$$

The reason why we use two AOMs to produce the frequency shift is that a single AOM cannot generate frequency shifts of order $1 \mathrm{MHz}$ or less as required. The velocity $v$ reported by the LDV is related by the Doppler equation with a frequency shift of $2 \delta$ and the wavelength of the laser by the relationship:

$$
v=1 / 2 \lambda(2 \delta)=\lambda \delta
$$

where the laser wavelength $\lambda=632.81 \mathrm{~nm}$ [7].

\section{RESULTS}

The experimental results that we report were obtained using [8] a commercially available LDV system that includes a Polytec OFV-503 Sensor
Head, a OFV-5000 Vibrometer Controller, and a Polytec Data Management System that were interfaced with the design shown in Figure 1. The AOMs (Brimrose TEF-110-50-633) were driven by two National Instruments PXIe-5650 sinusoidal radio frequency (RF) signal generators. An Agilent 3458A Digital Multimeter was also used to measure root mean squared (RMS) voltage for sinusoidal excitations. The signals from the RF signal generators were amplified using Mini-Circuits ZHL-2010+ RF amplifiers connected to each of the AOMs. The base frequency $f$ for our results was selected to be $110 \mathrm{MHz}$, corresponding to the center frequency of the AOMs. The zero frequency (DC) and transient velocity readings that we report were obtained using the Polytec Data Management System. The RMS readings that we report were obtained using the RMS multimeter.

\subsection{Results for fixed frequency shift}

Figure 2 shows the relationship between the frequency shift $\delta$, the reported velocity from the LDV, and the calculated velocity using the Doppler equation (2). These results were obtained with vibrometer controller set to VD-09 with a corresponding amplification factor of $0.5 \mathrm{~m} / \mathrm{s} / \mathrm{V}$. 


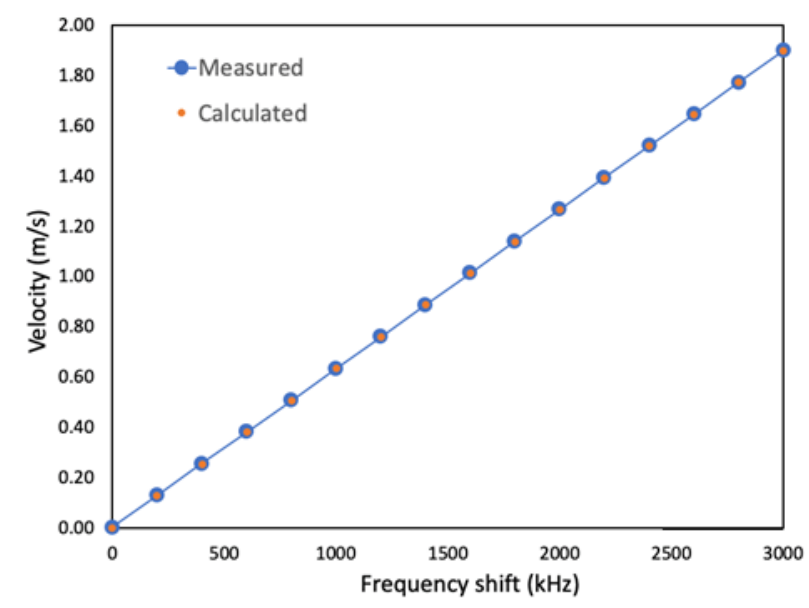

Figure 2: Plot of the velocity reported by the LDV as a function of frequency shift $\delta$ in blue and the corresponding calculated values using the Doppler equation (2).

The resulting dc voltage was sampled 2048 times at 204800 samples/s with no filtering using the Polytec Data Management System. The data were averaged, and the standard deviation was determined. The LDV exhibited a $0.0012 \mathrm{~m} / \mathrm{s}$ offset when directed onto a non-moving surface without the AOMs in the beam path as well as when the frequency shift $\delta$ was set to zero. This offset was subtracted from the measured results depicted in the figure.

The data in Figure 2 is replotted in Figure 3 in terms of the percent difference between the velocity reported by the LDV (with the offset subtracted) and the calculated velocity from the Doppler shift equation (2). The data show a maximum percent difference of $\pm 0.04 \%$ over the frequency range that was tested.

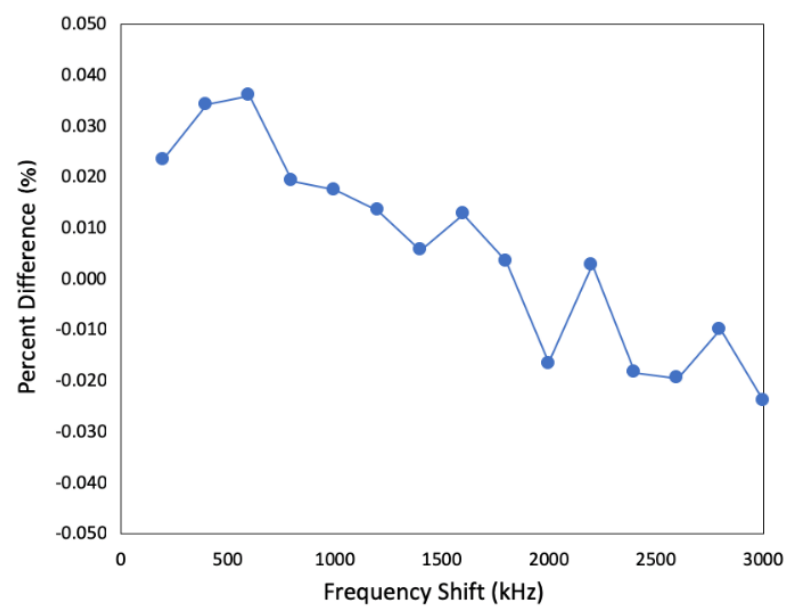

Figure 3: Percent difference between the velocity reported by the LDV (with the offset subtracted) and the calculated velocity using the Doppler shift equation (2). The manufacturer of the LDV reports a $1 \%$ uncertainty in their instrument specifications while the frequency shift that we can produce with the signal generators is orders of magnitude smaller in uncertainty.

\subsection{Results for sinusoidal excitation}

In this experiment the $2^{\text {nd }} A O M$ was excited using the National Instruments PXIe-5650 RF signal generator with a sinusoidal frequency modulation to create a synthesized vibration measurement. The goal of this experiment was to characterize the bandwidth of the LDV system. In this experiment, the root mean squared (RMS) voltage from the analog output of the Polytec OFV5000 Vibrometer Controller was measured using the RMS multimeter and converted to RMS velocity using the VD-09 gain factor of $0.5 \mathrm{~m} / \mathrm{s} / \mathrm{V}$. The sinusoidal modulation at the $110 \mathrm{MHz}$ base frequency was swept from $100 \mathrm{~Hz}$ to $3 \mathrm{MHz}$. Figure 4 shows that the LDV Vibrometer Controller has a uniform response up to $1 \mathrm{MHz}$ and drops off beyond that frequency.

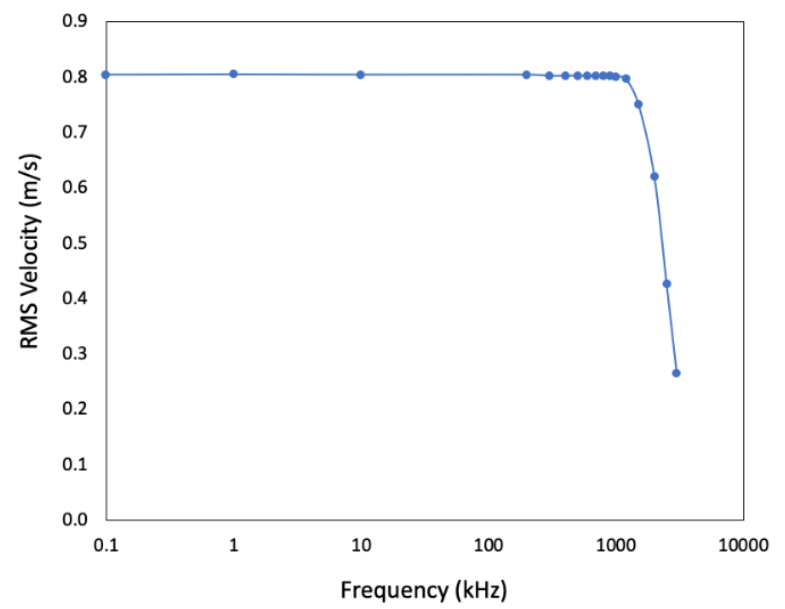

Figure 4: Frequency response of the LDV using sinusoidal frequency modulation of the $2^{\text {nd }} \mathrm{AOM}$ to create a synthesized vibration measurement condition.

\subsection{Results for velocity step function excitation}

In this experiment the $2^{\text {nd }} A O M$ was excited using an HP 83650B $10 \mathrm{MHz}$ to $50 \mathrm{GHz}$ RF Swept Signal Generator to provide a capability to frequency modulate an arbitrary analog signal. An Agilent 33250A Arbitrary Waveform Generator signal generator was used to produce a $1 \mathrm{~Hz}$ square wave alternating from $0 \mathrm{mV}$ to $300 \mathrm{mV}$ for frequency modulation to simulate a step function for synthesized velocity. The analog velocity signal from the Vibrometer Controller was digitized using the Polytec Data Management System set at its maximum sampling rate of 204800 samples/s. The resulting response shown in Figure 5 includes the effects of the LDV as well as the digital acquisition system, which would be expected to have a maximum bandwidth of $102400 \mathrm{~Hz}$. 


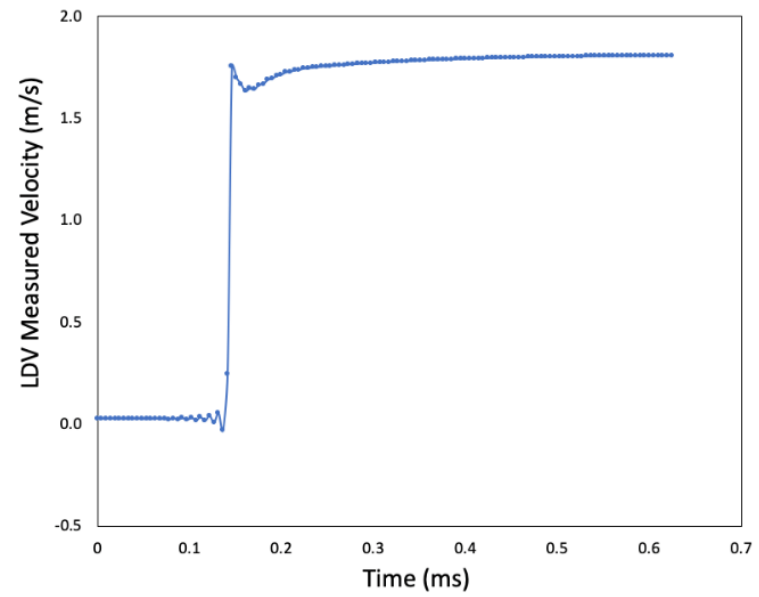

Figure 5: Response of the LDV system to a synthesized velocity step produced by frequency modulation of a $1 \mathrm{~Hz}$ square wave on the $2^{\text {nd }} A O M$. The time depicted on the $\mathrm{x}$-axis has been magnified to observe the transient of the velocity step.

\section{SUMMARY}

Our results show that this approach is immediately useful as a tool for characterizing DC, sinusoidal steady state, and transient response of an LDV as a system as a whole, together with its data acquisition and control electronics and amplifiers. The DC response we reported exhibited a maximum of $\pm 0.04 \%$ difference between the measured value and the calculated value based on the Doppler equation, which is in good agreement to what we had reported earlier using shock excitations and total distance travelled [5], and is well within the $1 \%$ accuracy specified by the manufacturer. The LDV system bandwidth of $1 \mathrm{MHz}$ determined by sinusoidal excitations is in good agreement to what the manufacturer specifies. Lastly, the velocity step function experiment serves as an example that it is possible to create complex velocity profiles to test the response of the LDV together with its data acquisition, control electronics, and amplifiers. Our future work is focused on further improvements on the system design and carrying out a full uncertainty analysis. One improvement that we envision in the design is measure the frequency shift $\delta$ with a photodiode rather than reading it from the signal generators driving the AOMs. This could capture any offsets or fluctuations between the signal generators and the light entering the LDV, e.g. due to refractive index fluctuation. We anticipate that after further investigation this method can be used as a tool for primary calibration of Laser Doppler Vibrometers.

\section{REFERENCES}

[1] ISO Standard 16063-41 Method for the calibration of vibration and shock transducers - Part 41 Calibration of laser vibrometers, ISO 1606341:2011(E).

[2] G. Siegmund, "Sources of Measurement Error in Laser Doppler Vibrometers and Proposal for Unified Specifications", Proc. of SPIE Vol. 7098, 70980Y-1, 2008.

[3] N. Vlajic, A. Chijioke, "Traceable dynamic calibration of force transducers by primary means," Metrologia 53, S136-S148, 2016.

[4] T. Bruns, F. Blume, A. Täubner, "Laser Vibrometer Calibration at High Frequencies using Conventional Calibration Equipment”, XIX IMEKO World Congress, Lisbon, Portugal, September 6-11, 2009.

[5] M. Gaitan, M. Afridi, J. Geist, "On the Calibration of Laser Heterodyne Velocimeters Using Shock Excitations and Total Distance Travelled", IMEKO XXII World Congress, Belfast, 2018.

[6] M. Afridi, J. Geist, M. Gaitan, "Primary Calibration of the Low Frequency Response of a Laser Heterodyne Velocimeter Used in a PendulumBased Shock Excitation System by SI Traceable Distance Measurement", J Res Natl Inst Stan, In Press.

[7] ISO Standard 16063-11 Methods for the calibration of vibration and shock transducers - Part 11 Primary vibration calibration by laser interferometry.

[8] Certain commercial equipment and instruments are identified in this article in order to describe the experimental procedure adequately. Such identification is not intended to imply recommendation or endorsement by the National Institute of Standards and Technology, nor is it intended to imply that the materials or equipment identified are necessarily the best available for the purpose. 